\title{
Radiographic features of Mycoplasma pneumoniae pneumonia: differential diagnosis and performance timing
} Naoyuki Miyashita*1, Tadaaki Sugiu ${ }^{1}$, Yasuhiro Kawai ${ }^{2}, K^{2}$ Kiko Oda $^{2}$,
Tetsuya Yamaguchi ${ }^{2}$, Kazunobu Ouchi ${ }^{2}$, Yoshihiro Kobashi ${ }^{1}$ and Mikio Oka ${ }^{1}$

\author{
Address: ${ }^{1}$ Division of Respiratory Diseases, Department of Medicine, Kawasaki Medical School, Kurashiki, Okayama, Japan and ${ }^{2}$ Department of \\ Pediatrics, Kawasaki Medical School, Kurashiki, Okayama, Japan \\ Email: Naoyuki Miyashita* - nao@med.kawasaki-m.ac.jp; Tadaaki Sugiu - tasugiu@med.kawasaki-m.ac.jp; \\ Yasuhiro Kawai - ykawai@med.kawasaki-m.ac.jp; Keiko Oda - koda@med.kawasaki-m.ac.jp; Tetsuya Yamaguchi - tetsuyaf@med.kawasaki- \\ m.ac.jp; Kazunobu Ouchi - kouchi@med.kawasaki-m.ac.jp; Yoshihiro Kobashi - yoshihiro@med.kawasaki-m.ac.jp; \\ Mikio Oka-moom@med.kawasaki-m.ac.jp \\ * Corresponding author
}

Published: 29 April 2009

BMC Medical Imaging 2009, 9:7 doi:10.1 186/147|-2342-9-7
Received: 19 January 2009

Accepted: 29 April 2009

This article is available from: http://www.biomedcentral.com/I47/-2342/9/7

(C) 2009 Miyashita et al; licensee BioMed Central Ltd.

This is an Open Access article distributed under the terms of the Creative Commons Attribution License (http://creativecommons.org/licenses/by/2.0), which permits unrestricted use, distribution, and reproduction in any medium, provided the original work is properly cited.

\begin{abstract}
Background: The Japanese Respiratory Society guidelines propose a differential diagnosis for atypical pneumonia and bacterial pneumonia using a scoring system for the selection of appropriate antibiotic. In order to improve this scoring system, the guidelines are seeking new specific parameter. The purpose of this study was to clarify the pattern of abnormalities with Mycoplasma pneumoniae pneumonia on chest computed tomography (CT) and whether the radiographic findings could distinguish $M$. pneumoniae pneumonia from Streptococcus pneumoniae pneumonia.
\end{abstract}

Methods: A retrospective review was performed of the CT findings of 64 cases and 68 cases where $M$. pneumoniae and $S$. pneumoniae, respectively, were the only pathogen identified by the panel of diagnostic tests used.

Results: Of the 64 patients with M. pneumoniae pneumonia, bronchial wall thickening was observed most frequently $(81 \%)$, followed by centrilobular nodules $(78 \%)$, ground-glass attenuation (78\%), and consolidation (6I\%). Bronchial wall thickening and centrilobular nodules were observed more often in $M$. pneumoniae patients than in $S$. pneumoniae patients $(p<0.000 \mathrm{I})$. The presence of bilateral bronchial wall thickening or centrilobular nodules was only seen in patients with $M$. pneumoniae pneumonia. Using the scoring system of the Japanese Respiratory Society guidelines and chest $C T$ findings, $97 \%$ of $M$. pneumoniae patients were suspected to be $M$. pneumoniae pneumonia without serology. When comparing the CT findings between early stage and progressed stage in the same patients with severe pneumonia, the radiographic features of early stage $M$. pneumoniae pneumonia were not observed clearly in the progressed stage.

Conclusion: The present results indicate that the diagnosis of $M$. pneumoniae pneumonia would appear to be reliable when found with a combination of bronchial wall thickening and centrilobular nodules in the CT findings. However, these CT findings are not observed in progressed severe $M$. pneumoniae pneumonia patients. 


\section{Background}

Community-acquired pneumonia (CAP) is a common infection, which is sometimes fatal. Despite substantial progress in therapeutic options, CAP remains a significant cause of morbidity and death worldwide (the fourth leading cause of death in Japan). Mycoplasma pneumoniae is one of the most frequent causes of respiratory tract infections, accounting for as many as $10-30 \%$ of all cases of CAP [1-3]. Epidemiological studies in Japan have demonstrated that the incidence of $M$. pneumoniae pneumonia is the second leading pathogen of CAP next to Streptococcus pneumoniae $[3,4]$. Serological testing is currently the most common tool for the diagnosis of M. pneumoniae infection. However, this test requires paired serum samples with a two to four week interval and provides only a retrospective diagnosis.

The etiological matter that most clearly differentiates Asia from Western countries is the frequency of drug-resistant $S$. pneumoniae, and especially the high-level of macrolideresistant $S$. pneumoniae [5-7]. Approximately half of $S$. pneumoniae cases show strong resistance to macrolides with minimum inhibitory concentrations greater than or equal to $128 \mu \mathrm{g} / \mathrm{mL}[5,7]$. Therefore, it is difficult to manage both bacterial and atypical pneumonias by medication with macrolide antibiotics as the first-choice drug. In addition, in recent years the incidence of fluoroquinolone-resistant $S$. pneumoniae has risen markedly and particularly in patients over 60 years of age $[7,8]$. Furthermore, fluoroquinolone-resistant Haemophilus influenzae has also emerged $[7,9]$. Therefore, the usage of fluoroquinolones as an empirical first choice drug should be limited to only selected patients who have co-morbid illnesses, are of an older age group, have used antibiotics recently, or have severe CAP to prevent an increase in the frequency of quinoline-resistant strains [4].

These facts indicate that it could be important to distinguish atypical pneumonia, especially $M$. pneumoniae pneumonia, from $S$. pneumoniae pneumonia for the empirical selection of antibacterial therapy. Thus, the Japanese Respiratory Society (JRS) CAP guidelines are on trial to differentiate between atypical pneumonia and bacterial pneumonia using a scoring system [4]. The guidelines have six parameters and criteria based on clinical features of $M$. pneumoniae pneumonia [10]. However, using this system there are still many cases where the guidelines cannot differentiate between $M$. pneumoniae pneumonia and $S$. pneumoniae pneumonia, and the JRS guidelines are seeking new specific parameters in order to improve this scoring system [4]. Some reports have focused on radiographic features using high-resolution computed tomography (HRCT) of M. pneumoniae pneumonia [11-15]. However, to our knowledge, there have been relatively few comparative studies that have investigated the radio- graphic findings of $M$. pneumoniae pneumonia versus $S$. pneumoniae pneumonia. The purpose of this study was to clarify the pattern of abnormalities with M. pneumoniae pneumonia on chest CT and whether the radiographic findings could distinguish $M$. pneumoniae pneumonia from $S$. pneumoniae pneumonia. Furthermore, this study also evaluated the performance of CT scans take at different stages of infection.

\section{Methods \\ Study population}

The study was retrospective and included adult patients with CAP who underwent HRCT. In all patients, CT images were obtained before treatment with antibiotics. Patients were seen at Kurashiki Daiichi Hospital, Kawasaki Medical School Kawasaki Hospital, and Kawasaki Medical School Hospital, Okayama, Japan, between April 2000 and December 2008. None of the patients were immunocompromised; namely patients with HIV infection, neutropenia secondary to chemotherapy, or patients on immunosuppressants; or patients with history of chronic lung diseases, from nursing homes, or patients with recent (<30 days) admission to hospital. The diagnosis was based on clinical signs and symptoms (cough, fever, productive sputum, dyspnea, chest pain, or abnormal breath sounds), and radiographic pulmonary abnormalities that were at least segmental and were not due to pre-existing or other known causes. All cases of pneumonia occurring more than three days after hospitalization were considered nosocomial and were excluded. The study protocol was approved by the Ethics Committee at Kawasaki Medical School.

\section{Microbiological laboratory tests}

Microbiological tests such as Gram stain, cultures, urinary antigen tests, and serological tests were performed as described previously $[3,16]$. Blood cultures and nasopharyngeal swab specimens were obtained from all patients on admission and, if pleural fluid and sputum were available, a Gram stain test and a quantitative culture were obtained. Sputum data were only evaluated when the Gram stain test revealed numerous leukocytes $(>25$ in a $\times$ 100 microscopic field) and few squamous epithelial cells $(<10$ in a $\times 100$ microscopic field). Certain invasive methods such as bronchoscopic examination were employed to obtain specimens in some patients after full explanation of the procedures. These specimens were also used for culturing of M. pneumoniae and Legionella species on pleuropneumonia-like organism broth and agar (70\% pleuropneumonia-like organism broth [Difco, Inc., Detroit, MI, USA] supplemented with $20 \%$ heat-inactivated horse serum, $10 \%$ fresh yeast extract [25\%], thallium acetate [final concentration $0.5 \mathrm{mg} / \mathrm{mL}$ ], and sterile penicillin G [final concentration 1,000 U/mL]) and buffered charcoalyeast extract alpha agar, respectively. Cultures for Chlamy- 
dophila pneumoniae and C. psittaci were performed using cycloheximide-treated HEp-2 cells grown in a 24-well cell culture plate [16]. All specimens were passed twice. Culture confirmation was done by fluorescent-antibody staining with C. pneumoniae and C. psittaci species-specific and genus-specific monoclonal antibodies. Bronchoscopic specimens were also used for polymerase chain reaction (PCR) of M. pneumoniae and chlamydial species. The M. pneumoniae-specific primers used for the PCR were from the DNA base sequence within the P1 cytadhesin gene, and amplification was performed as reported by Ramirez et al. [17]. The C. pneumoniae-specific and genusspecific primers used for PCR were from the DNA base sequence within the 53-kDa protein gene and major outer membrane protein gene, respectively, established in our laboratory. These assays were performed as described previously $[3,18]$.

Paired serum samples were collected at intervals of at least four weeks after onset. Complement fixation tests were done in all patients for antibodies to influenza A and B viruses, adenovirus, respiratory syncytial virus, cytomegalovirus, and parainfluenza virus types 1,2 , and 3 . Antibodies to $M$. pneumoniae were measured by a passive agglutination test (Serodia-Myco II kit, Fujirebio, Tokyo, Japan), Legionella species by a microagglutination test (detection of L. pneumophila serogroups 1 6, L. bozoemanii, L. dumoffii, L. gormanii, and L. micdadei), and Coxiella burnetii by an indirect immunofluorescence test. A microimmunofluorescence test was used for the titration of IgG and IgM antibodies against chlamydial species, using formalinized elementary bodies of C. pneumoniae KKpn-15, C. trachomatis $\mathrm{L} 2 / 434 / \mathrm{Bu}$, and C. psittaci Budgerigar-1 strains as antigens [16]. Rheumatoid factors were absorbed with GullSORB (Meridian Bioscience Inc., OH, USA) before IgM titrations. In addition to serology, culturing, and/or PCR, a urinary antigen test (Binax NOW, Binax Inc. Portland, ME, USA) was used for the detection of S. pneumoniae and L. pneumophila.

\section{Criteria for the determination of microbial etiology}

Microbial etiology was classified as "definitive", "presumptive", or "unknown" as reported previously [3]. Bacteria were considered to be definitive causative agents when isolated from blood or pleural fluid cultures. The results of sputum cultures were considered in combination with Gram stain findings. An organism showing heavy $\left(\geq 10^{7} \mathrm{cfu} / \mathrm{mL}\right)$ or moderate $\left(10^{6} \mathrm{cfu} / \mathrm{mL}\right)$ growth of a predominant bacterium on a sputum culture was considered to be a presumptive pathogen. Any microorganism isolated from bronchoscopic specimens was considered to be a presumptive pathogen when its concentration reached $>10^{5} \mathrm{cfu} / \mathrm{mL}$ in quantitative culture. If M. pneumoniae or Legionella species were isolated from a specimen, that specimen was considered to be a definitive pathogen even if the culture showed little growth. L. pneumophila and S. pneumoniae were considered to be presumptive agents when the urinary antigen test was positive. For serological tests, a four-fold rise in the antibody titer level between paired sera was considered definitive. Acute C. pneumoniae or C. psittaci infection was defined as IgM $\geq 1: 32$ or a four-fold rise in IgG or IgM titer between acute and convalescent serum samples.

\section{Chest CT findings}

CT examinations were performed using a Hi-Speed Advantage scanner (General Electric Medical Systems, Milwaukee, WI, USA), Hi-Speed LX/i Advantage (General Electric Medical Systems), or a Light Speed Ultra (General Electric Medical Systems). All scans were obtained during suspended end inspiration with the patient in a spine position. HRCT was performed with 1-mm collimation at 10-mm intervals. Images were obtained at lung (level -700 $\mathrm{HU}$; width, 1,500 HU) and mediastinal (level 20-40 HU; width, $400 \mathrm{HU}$ ) levels. The time between clinical onset of pneumonia (fever and/or respiratory symptoms) and CT ranged from 1 to 10 days (mean, 5.1 days) for M. pneumoniae pneumonia and from 1 to 12 days (mean, 5.2 days) for $S$. pneumoniae pneumonia. Contrast material was used in 12 patients with M. pneumoniae or S. pneumoniae pneumonia.

Three chest radiologists (16, 12 and 10 years experience, respectively), who were blinded to the diagnoses, retrospectively and independently assessed the presence of consolidation, ground-glass attenuation, centrilobular nodules, thickening of the bronchial wall, reticular or linear opacity, pleural effusion, and lymphadenopathy. Readers also evaluated if the pneumonia was unilateral or bilateral and identified the opacity pattern of pneumonia. In addition, it was recorded which lobe of the lung was involved.

Consolidation was defined as air-space opacification with obscuration of the underlying vasculature. Ground-glass attenuation was defined as mildly increased attenuation without obscuration of the underlying vasculature. A centrilobular nodule was defined as a nodule identified around the peripheral pulmonary arterial branches or 3 to $5 \mathrm{~mm}$ away from the pleura, interlobular septa, or pulmonary veins. Bronchial wall thickening was defined as the thickening identified widespread areas not close to areas of ground-glass attenuation and/or consolidation. Interlobular septa thickening, intralobular interstitial thickening, and areas of irregular linear opacity were all classified as reticular or linear opacity. The reticular framework in ground-glass attenuation that is described as crazy-paving appearance was not classified as an area of reticular or linear opacity. Mediastinal lymphadenopathy was judged to be present when the minimal diameter of a lymph node 
was larger than $10 \mathrm{~mm}$. Hilar lymphadenopathy was judged to be present only if the maximum diameter of the ipsilateral hilum exceeded that of the contralateral hilum by 1.5 -fold or more. The final decisions for the presence of each finding and the opacity pattern for each case were reached by the consensus of the three radiologists.

\section{Differential diagnosis in JRS guidelines}

The JRS guidelines include six parameters and criteria based on underlying conditions, clinical symptoms, and laboratory findings by multiple regression analysis [10]. These parameters are; 1 ) under 60 years of age, 2) no or minor co-morbid illness, 3 ) the patient has stubborn cough, 4) the patient has poor chest auscultatory findings, 5) no sputum or identified etiological agent by rapid diagnostic tests, and 6) a peripheral white blood cell (WBC) count below $10,000 / \mathrm{mm}^{3}$. When there is a correlation of more than four parameters out of six, then the guidelines recommend the use of macrolides or tetracyclines for suspected M. pneumoniae pneumonia [4]. If these criteria are not met, the guidelines recommend the use of $\beta$-lactams for suspected S. pneumoniae pneumonia [4].

\section{Statistical analysis}

Statistical analysis was performed using Stat View version 5.0. (SAS Institute Inc, Cary, NC, USA). The incidence of underlying conditions, clinical findings, and radiographic findings were analyzed using Fisher's Exact test. Mean age of patients and laboratory data were compared using Student's $t$ test. For each radiological finding, kappa values were calculated between the readers.

\section{Results}

Five hundred and sixty-five patients were enrolled in this study. A microbiological diagnosis was established in 298 patients. Among all CAP cases, there were 64 cases and 68 cases where M. pneumoniae and S. pneumoniae, respectively, was the only pathogen identified by the panel of diagnostic tests used. Cases of pneumonia mixed with other microorganisms were excluded in this study. All $M$. pneumoniae cases demonstrated four-fold antibody seroconversion (10 cases were culture positive).

Table 1 shows the clinical characteristics of pneumonia patients on admission to hospital. The mean age of the patients with $M$. pneumoniae pneumonia was significantly lower than that of those with $S$. pneumoniae pneumonia ( $p$ $<0.0001)$. The frequency of a co-morbid illness with $M$. pneumoniae pneumonia was significantly lower than with $S$. pneumoniae pneumonia $(p<0.0001)$. The most common co-morbid illness was diabetes mellitus (M. pneumoniae 6, S. pneumoniae 10), followed by cardiovasucular diseases (M. pneumoniae 3, S. pneumoniae 7), chronic liver disease (M. pneumoniae 3, S. pneumoniae 6), and cerebrovascular diseases (M. pneumoniae $1, S$. pneumoniae 5). The
Table I: Clinical characteristics in patients with Mycoplasma pneumoniae pneumonia and Streptococcus pneumoniae pneumonia on admission*

\begin{tabular}{llll}
\hline Characteristics & M. pneumoniae & S. pneumoniae & $p$ value \\
\hline Number & 64 & 68 & \\
Mean age (range), years & $36.6(18-69)$ & $61.8(19-86)$ & $<0.000$ I \\
$\begin{array}{l}\text { Male: Female } \\
\text { Co-morbid illness }\end{array}$ & $34: 30$ & $42: 26$ & 0.379 I \\
PSI risk classes** & $15(23)$ & $33(48)$ & $<0.000$ I \\
$\quad$ I - III & $41(64)$ & $29(43)$ & 0.0154 \\
IV & $23(36)$ & $39(57)$ & \\
V & 0 & 0 & \\
WBC mean $(/ \mu \mathrm{L})$ & 7,400 & 13,800 & $<0.000$ I \\
\hline
\end{tabular}

*Data represent the numbers of patients, and numbers in parentheses are percentages.

**PSI, pneumonia severity index.

mean WBC count on admission was significantly lower in patients with $S$. pneumoniae pneumonia $(p<0.0001)$. These results were almost identical to the findings of previous studies $[16,19]$.

The kappa value between the readers was 0.607 for consolidation, 0.552 for ground-glass attenuation, 0.746 for centrilobular nodules, 0.564 for thickening of the bronchial wall, 0.587 for reticular or linear opacity, 0.882 for pleural effusion, and 0.618 for lymphadenopathy. These values indicated fair to good interreader agreement.

Chest CT findings of the patients with $M$. pneumoniae pneumonia and $S$. pneumoniae pneumonia are listed in Table 2. Of the 64 patients with M. pneumoniae pneumonia, bronchial wall thickening was observed most frequently ( $81 \%)$, followed by centrilobular nodules (78\%), ground-glass attenuation (78\%), and consolidation (61\%) (Figures 1 and 2). Reticular or linear opacities, pleural effusion, and lymphadenopathy were also observed, but at low frequencies. Among the CT findings, bronchial wall thickening and centrilobular nodules were observed more often in M. pneumoniae patients than in $S$. pneumoniae patients $(p<0.0001)$. Air-space consolidation was often seen in both pneumonias but as a non-specific finding ( $p=0.0618$ ) (Figure 3). Bronchial wall thickening or centrilobular nodules, which were considered to be a spectrum of HRCT findings for bronchopneumonia, were seen in $59(92 \%)$ patients with M. pneumoniae pneumonia, and in $20(29 \%)$ patients with $S$. pneumoniae pneumonia, which was a statistically significant difference $(p<$ 0.0001 ). The presence of bilateral bronchial wall thickening or centrilobular nodules was only seen in patients with M. pneumoniae pneumonia.

The frequency of involvement in each lobe was not statistically different between patients with $M$. pneumoniae pneumonia and S. pneumoniae pneumonia (Table 3). 


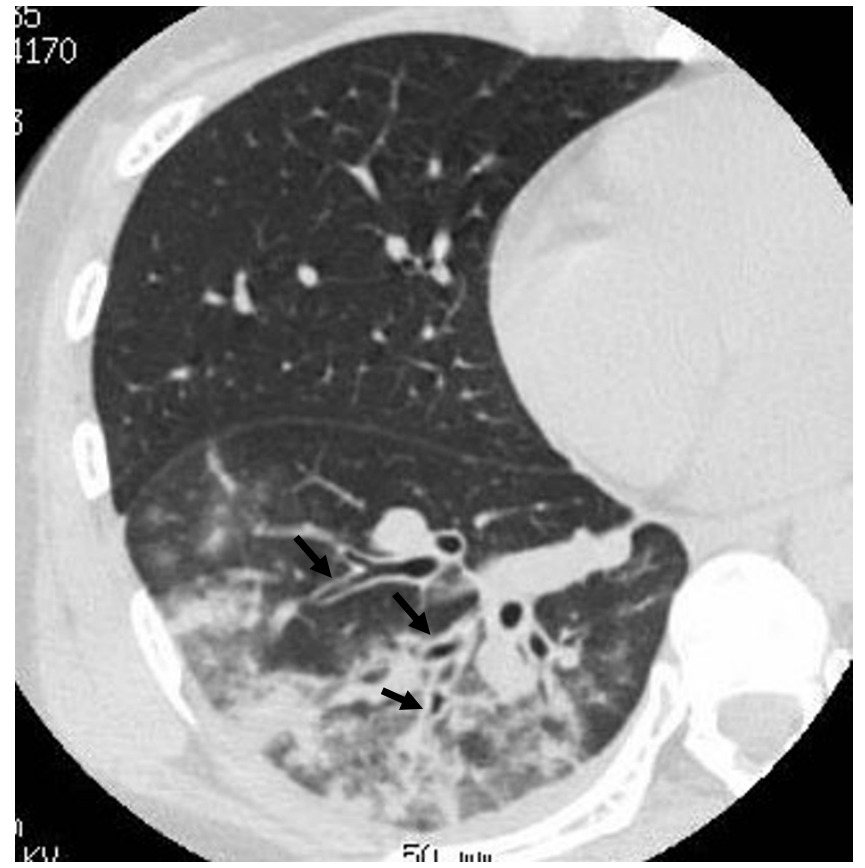

Figure I

30-year-old woman with $M$. pneumoniae pneumonia. CT shows bronchial wall thickening (arrows). Lobular areas of consolidation and ground-glass attenuation are also seen.

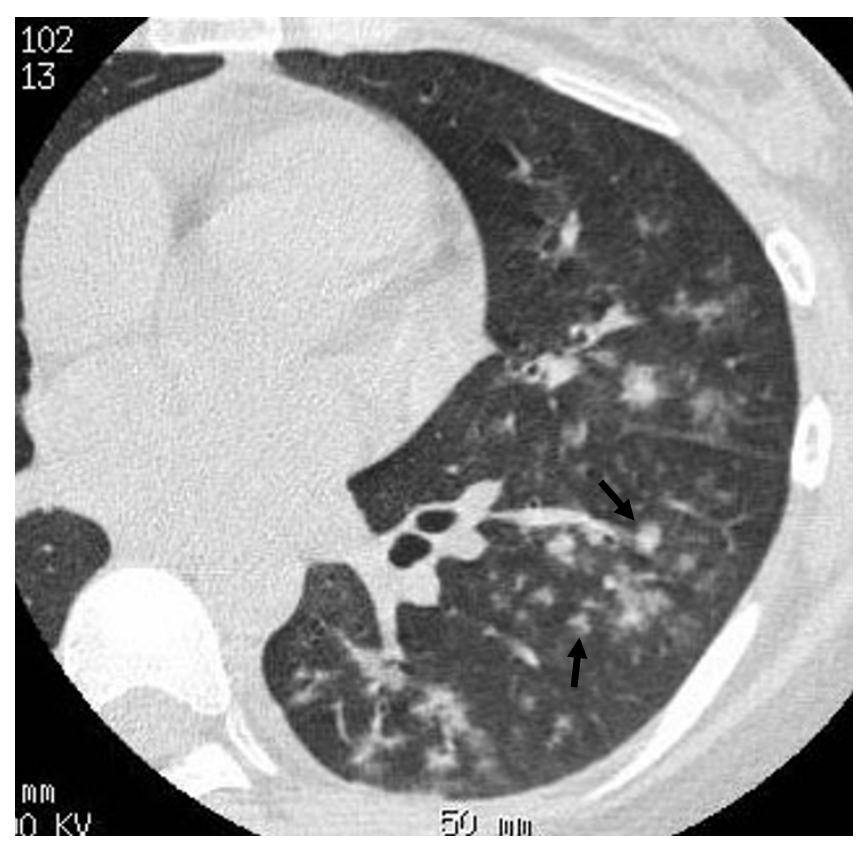

Figure 2

24-year-old man with $M$. pneumoniae pneumonia. CT shows centrilobular nodules (tree-in-bud appearance, arrows). Bronchial wall thickening is also seen.

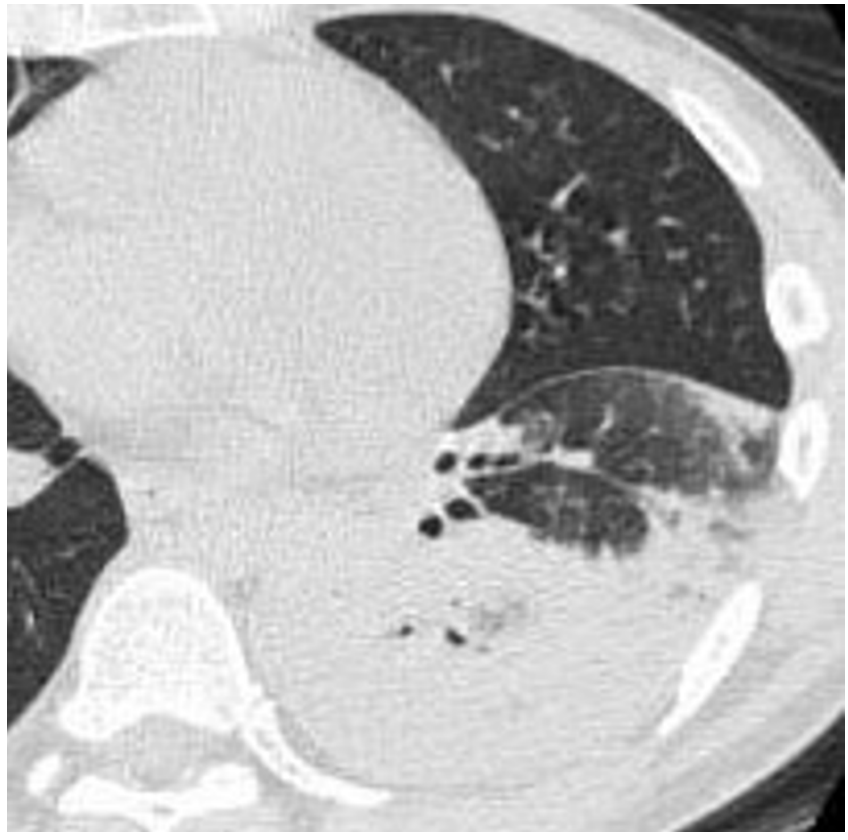

Figure 3

39-year-old man with S. pneumoniae pneumonia. CT shows air-space consolidation in left lower lobe.

However, the frequency of involvement of more than one lobe was seen more often in patients with $M$. pneumoniae pneumonia than in patients with $S$. pneumoniae pneumonia $(p=0.0005)$.

Using the scoring system of the JRS guidelines, 12 of 64 patients with $M$. pneumoniae pneumonia were judged as bacterial pneumonia and 10 of 68 patients with S. pneumoniae pneumonia were judged as atypical pneumonia. Of the 12 patients with M. pneumoniae pneumonia who were judged as having bacterial pneumonia, 10 patients showed the radiographic features of $M$. pneumoniae pneumonia with the combination of bronchial wall thickening and centrilobular nodules. In contrast, 10 patients with $S$.

Table 2: Chest CT findings of the patients with Mycoplasma pneumoniae pneumonia and Streptococcus pneumoniae pneumonia on admission*

\begin{tabular}{llll}
\hline Findings & $\begin{array}{l}\text { M. pneumoniae } \\
(\mathrm{n}=64)\end{array}$ & $\begin{array}{l}\text { S. pneumoniae } \\
(\mathrm{n}=68)\end{array}$ & $\mathrm{p}$ value \\
\hline Consolidation & $39(61)$ & $52(76)$ & 0.0618 \\
Ground-glass attenuation & $50(78)$ & $32(47)$ & 0.0003 \\
Centrilobular nodules & $50(78)$ & $15(22)$ & $<0.000 \mathrm{I}$ \\
Bronchial wall thickening & $52(81)$ & $13(19)$ & $<0.000 \mathrm{I}$ \\
Reticular or linear opacity & $17(27)$ & $22(32)$ & 0.5675 \\
Pleural effusion & $13(20)$ & $17(25)$ & 0.5409 \\
Lymphadenopathy & $15(23)$ & $13(19)$ & 0.6707
\end{tabular}

* Data represent the numbers of patients and numbers in parentheses are percentages. 
Table 3: Frequency of lobe involvement*

\begin{tabular}{llll}
\hline Parameter & $\begin{array}{l}\text { M. pneumoniae } \\
(\mathrm{n}=64)\end{array}$ & $\begin{array}{l}\text { S. pneumoniae } \\
(\mathrm{n}=68)\end{array}$ \\
\hline Lobe & & & \\
$\quad$ Upper & $33(55)$ & $30(44)$ & 0.2960 \\
Middle & $31(48)$ & $22(32)$ & 0.0759 \\
$\quad$ Lower & $51(80)$ & $47(69)$ & 0.2319 \\
More than one lobe involved & $46(72)$ & $31(47)$ & 0.0005 \\
\hline
\end{tabular}

* Data represent numbers of patients and numbers in parentheses are percentages.

pneumoniae pneumonia who were judged as atypical pneumonia did not show the radiographic features of $M$. pneumoniae pneumonia.

During the study period, five of 64 patients with M. pneumoniae pneumonia progressed severe pneumonia with respiratory failure requiring mechanical ventilation (progressed stage). In these five patients, chest CT was performed in both the non-progressed (mild to moderate severity pneumonia without respiratory failure) and the progressed stage. The time between clinical onset of pneumonia (fever and/or respiratory symptoms) and CT (progressed stage) ranged from 8 to 15 days (mean, 10.9 days). All five patients showed the radiographic features of M. pneumoniae pneumonia, such as bronchial wall thickening and centrilobular nodules on admission. However, at the progressed stage these patients showed bilateral diffuse infiltrates and did not show the radiographic features of $M$. pneumoniae pneumonia that was observed in the non-progressed stage (Figure 4). There were no any differences in terms of clinical characteristics on admission between the five patients with progressed severe pneumonia and the remaining patients.

\section{Discussion}

One of the basic policies and main purposes of the JRS CAP guidelines include the prevention of bacterial resistance because of the high frequency of drug-resistant respiratory pathogens among Asian countries [4]. As shown in Table 1, there are many clinical differences between $M$. pneumoniae pneumonia and $S$. pneumoniae pneumonia. These results were similar to those described in previous reports $[16,19]$. Thus, the JRS guidelines propose a differential diagnosis for atypical pneumonia, especially $M$. pneumoniae pneumonia, and bacterial pneumonia, predominantly $S$. pneumoniae pneumonia, for the selection of appropriate antibiotic management of CAP [4]. Usually, chest radiography is the first imaging technique obtained for the evaluation of acute respiratory symptoms. However, the patterns of presentation of $M$. pneumoniae pneumonia on chest radiography are nonspecific, consisting of patchy areas of airspace consolidation, reticular interstitial infiltrates, or both $[10,19]$. Thus, the JRS guidelines excluded chest radiographic findings from a differential table when the guidelines were updated [4]. In contrast, CT images can more accurately provide detailed information about the lung parenchyma than routine chest radiography. Reittner et al. compared the radiographic and chest CT findings of $M$. pneumoniae pneumonia and concluded that the lobular distribution, centrilobular involvement, and interstitial abnormalities in M. pneumoniae pneumonia are often difficult to recognize on radiographs but these features can usually be seen on CT [20].

In this study, the CT findings of M. pneumoniae pneumonia were evaluated and compared it with $S$. pneumoniae pneumonia, which is the leading pathogen of CAP worldwide. The most important differences between M. pneumoniae and $S$. pneumoniae pneumonias were the frequency of bronchial wall thickening and centrilobular nodules. These findings were observed more often in M. pneumo-

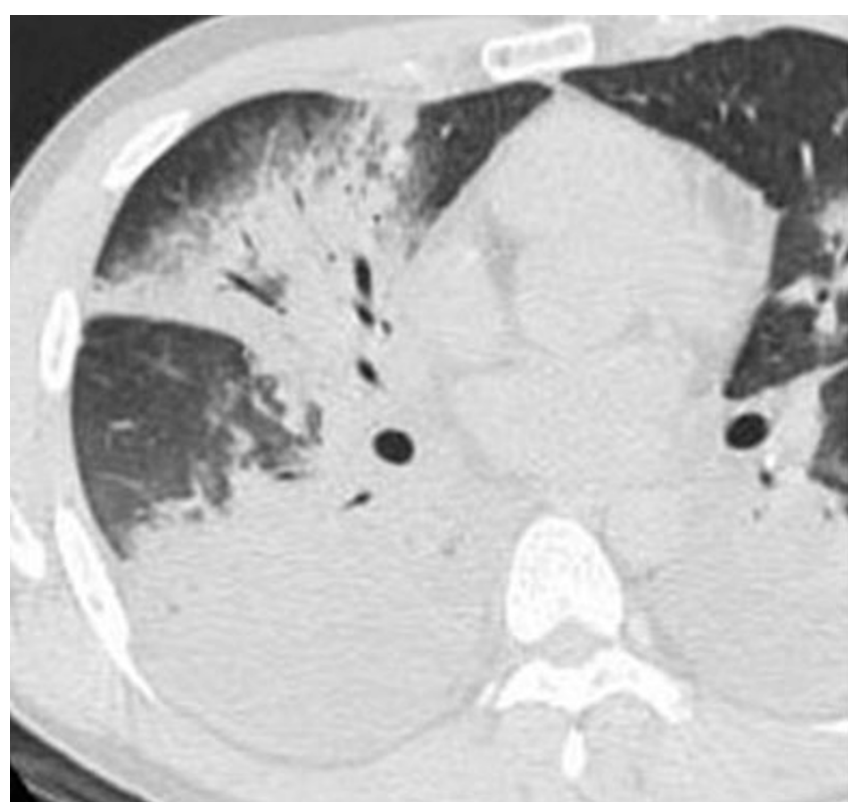

Figure 4

3 I-year-old man with severe $M$. pneumoniae pneumonia with respiratory failure requiring mechanical ventilation. CT shows bilateral air-space consolidation and pleural effusion. 
niae patients than in $S$. pneumoniae patients $(p<0.0001)$. Furthermore, the presence of bilateral bronchial wall thickening or centrilobular nodules may be highly suggestive for M. pneumoniae pneumonia. Nambu et al. reported that the difference in frequency of bronchovascular bundle thickening (bronchial wall thickening) and peribronchovascular or centrilobular nodules between $M$. pneumoniae and $S$. pneumoniae was statistically significant $(p=0.004)[15]$. Air-space consolidation with or without centrilobular or peribronchovascular nodules were often seen in both pneumonias and were nonspecific. Reittner et al. also reported that centrilobular nodules were detected more frequently in $M$. pneumoniae pneumonia $(86 \%)$ than in bacterial pneumonias $(17 \%, p<0.01)$ [20]. The finding of bronchial wall thickening was not evaluated in that study. These data together with the present results suggest that $M$. pneumoniae pneumonia may be differentiated from S. pneumoniae pneumonia using CT findings with the combination of bronchial wall thickening and centrilobular nodules.

To confirm whether CT findings can differentiate M. pneumoniae pneumonia from $S$. pneumoniae pneumonia, patients with M. pneumoniae pneumonia who were suspected as having bacterial pneumonia were evaluated by the scoring system of the JRS guidelines. Of these, $83 \%$ patients could be differentiated from $S$. pneumoniae pneumonia by radiographic features. In contrast, all patients with S. pneumoniae pneumonia who were suspected as atypical pneumonia did not show the radiographic features of M. pneumoniae pneumonia. Thus, the diagnosis of M. pneumoniae pneumonia appeared to be reliable when found with the combination of bronchial wall thickening and centrilobular nodules in the CT findings.

The M. pneumoniae organism attaches to cilia through the P1 protein and multiplies in the respiratory epithelial layer [1]. Attachment to epithelial cilia is essential for $M$. pneumoniae infection, through to progression and manifestation as bronchial wall thickening. Histopathologically, it is characterized by acute cellular bronchiolitis with edematous and ulcerative lesions of the bronchial walls and by peribronchial and perivascular interstitial opacities containing lymphocytes, plasma cells, and macrophages [21-23]. In contrast, $S$. pneumoniae may not have this biological capacity to directly infect the alveolar lumen, thereby allowing pneumonia to progress [24]. Although S. pneumoniae can occasionally damage the respiratory epithelium directly, it rarely produces ongoing disease in segmental bronchi. The main site of inflammation may reflect the CT findings.

Pneumonia due to M. pneumoniae is usually mild, but some cases are known to develop into severe, life-threatening pneumonia with respiratory failure and acute respi- ratory distress syndrome $[18,25,26]$. This study compared the CT findings between the early stage (mild to moderate severity) and progressed stage (severe pneumonia with respiratory failure) of $M$. pneumoniae pneumonia. Unfortunately, all patients with progressed pneumonia did not show the typical radiographic features of $M$. pneumoniae pneumonia. For further evaluation of CT findings at the progressed stage, 13 severe $M$. pneumoniae pneumonia with respiratory failure were evaluated that were described in a previous report [18]. These severe cases showed the same CT findings of the above progressed cases and could not be differentiated from $S$. pneumoniae pneumonia.

The limitation of the present study is the lack of inclusion of pneumonia due to other common microorganisms such as C. pneumoniae or Haemophilus influenzae. Because C. pneumoniae pneumonia is usually mild and self-limiting, it is treated in an outpatient setting without CT scanning [3]. H. influenzae is usually detected from patients with chronic lung diseases such as bronchiectasis, diffuse panbronchiolitis, or chronic obstructive pulmonary disease. Thus, it is difficult to evaluate the precise CT findings in $H$. influenzae pneumonia. Another limitation of the present study is that it excluded patients with chronic lung diseases and severe pneumonia for the precise evaluation of CT findings. These diseases could influence CT findings. In addition, M. pneumoniae usually infects young adults with no or mild co-morbid illnesses $[16,18,19]$. A further weakness of this study is its retrospective design and small sample size, which are characteristic of such preliminary studies. Thus, further studies are necessary to determine whether CT findings could differentiate the various microorganisms with or without chronic lung diseases. However, the usefulness of CT findings is limited to hospitals with CT scanners. The guidelines are aimed at general practitioners and non-specialized doctors and were selected to allow easy differentiation of CAP in an outpatient setting without special examinations [4]. Thus, the JRS guidelines may not recommend the utilization of chest CT routinely.

\section{Conclusion}

The main purpose of the present study was to identify a means of rapidly distinguishing $M$. pneumoniae pneumonia from $S$. pneumoniae pneumonia in daily clinical practice without waiting for serological results. The results indicate that the combination of bronchial wall thickening and centrilobular nodules on chest CT is most suggestive of M. pneumoniae pneumonia. The presence of bilateral bronchial wall thickening or centrilobular nodules was highly suggestive of $M$. pneumoniae pneumonia.

\section{Competing interests}

The authors declare that they have no competing interests. 


\section{Authors' contributions}

$\mathrm{NM}$ and $\mathrm{KO}$ conceived the study and participated in its design and coordination. NM, TS, KO, YK, and MO collected and managed the data, including quality control. $\mathrm{YK}, \mathrm{KO}$, and TY carried out the microbiological laboratory tests. NM and KO drafted the manuscript, and all authors contributed substantially to its revision. All the authors read and approved the final manuscript.

\section{Acknowledgements}

We gratefully acknowledge the members of the Kurashiki Chest Conference Group for advising and reading the chest $C T$ findings. This work was supported by MEXT KAKENHI (1959II 90 and 21591304) and Project Research Grants from Kawasaki Medical School (13-40I, 14-402, 15-405A, 16-405M, 17-402M, 18-40I, 19-402M, 20-4030).

\section{References}

I. Waites KB, Talkington DF: Mycoplasma pneumoniae and its role as a human pathogen. Clin Microbiol Rev 2004, 17:697-728.

2. Blasi F: Atypical pathogens and respiratory tract infections. Eur Respir J 2004, 24: 17I-8I.

3. Miyashita N, Fukano H, Mouri K, Fukuda M, Yoshida K, Kobashi Y, Niki Y, Oka M: Community-acquired pneumonia in Japan: a prospective ambulatory and hospitalized patient study. J Med Microbiol 2005, 54:395-400.

4. The committee for the JRS guidelines in management of respiratory infections: The JRS guidelines for the management of community-acquired pneumonia in adults [in Japanese]. Edited by: Matsushima T, Kohno S, Saito A, Nakata K, Yamaguchi K, Watanabe A, Aoki N, Goto H, Suzuki K, Sekizawa K, Souma K, Nagatake T, Mikasa K, Miyashita N, Ishida T, Tomono K, Nakahama C. The Japanese Respiratory Society. Tokyo, Japan; 2005.

5. Niki Y, Hanaki H, Yagisawa M, Kohno S, Aoki N, Watanabe A, Sato J, Hattori R, Koashi N, Kozuki T, Maruo A, Morita K, Ogasawara K Takahashi Y, Watanabe J, Takeuchi K, Takahashi M, Takeda H, Ikeda H, Kaneda H, Niitsuma K, Saito M, Koshiba S, Kaneko M, Itabashi S, Miki M, Nakanowatari S, Honda Y, Chiba J, Takahashi H, Utagawa M, Kondo T, Kawana A, Konosaki $\mathrm{H}$, Aoki $Y$, Chonabayashi N, Ueda $\mathrm{H}$, Sugiura $H$, Ichioka M, Goto $H$, Aoshima M, Okazaki M, Ozawa T, Horiuchi F, Yoshida T, Tsukada H, Kobayashi S, Yoshikawa H, Imai Y, Aoki N, Honma Y, Yoshida K, Takaya M, Kurokawa Y, Kuwabara M, Fujiue Y, Ishimaru T, Matsubara N, Kawasaki Y, Tokuyasu H, Masui K, Shimizu E, Yoneda K, Negayama K, Ueda N, Ishimaru M, Nakanishi Y, Fujita M, Honda J, Kadota J, Hiramatsu K, Aoki Y, Nagasawa Z, Suga M, Muranaka H, Kohno S, Yanagihara K, Fujita J, Tateyama M, Totsuka $\mathrm{K}$, Japanese Society of Chemotherapy: The first nationwide surveillance of bacterial respiratory pathogens conducted by the Japanese Society of Chemotherapy. Part I: a general view of antibacterial susceptibility. J Infect Chemother 2008, 1 4:279-90.

6. Song JH, Jung SI, Ko KS, Kim NY, Son JS, Chang HH, Ki HK, Oh WS, Suh JY, Peck KR, Lee NY, Yang Y, Lu Q, Chongthaleong A, Chiu CH, Lalitha MK, Perera J, Yee TT, Kumarasinghe G, Jamal F, Kamarulzaman A, Parasakthi N, Van PH, Carlos C, So T, Ng TK, Shibl A: High prevalence of antimicrobial resistance among clinical Streptococcus pneumoniae isolates in Asia (an ANSORP study). Antimicrob Agents Chemother 2004, 48:210I-7.

7. Inoue M, Lee NY, Hong SW, Lee K, Felmingham D: PROTEKT 1999-2000: a multicentre study of the antibiotic susceptibility of respiratory tract pathogens in Hong Kong, Japan and South Korea. Int J Antimicrob Agents 2004, 23:44-5I.

8. Yokota S, Sato K, Yoshida S, Fujii N: Molecular epidemiology of fluoroquinolone-resistant Streptococcus pneumoniae in Japan. Kansenshogaku Zasshi 2004, 78:428-34. In Japanese

9. Yokota S, Ohkoshi Y, Sato K, Fujii N: Emergence of fluoroquinolone-resistant Haemophilus influenzae strains among elderly patients but not among children. J Clin Microbiol 2008 46:36I-5.

10. Ishida T, Miyashita N, Nakahama C: Clinical differentiation of atypical pneumonia using Japanese guidelines. Respirology 2007, 12:104-10.
II. Tanaka N, Matsumoto T, Kuramitsu T, Nakai H, Ito K, Uchisako H, Miura G, Matsunaga N, Yamakawa $\mathrm{K}$ : High resolution $\mathrm{CT}$ findings in community-acquired pneumonia. J Comput Assist Tomogr 1996, 20:600-8.

12. Reittner P, Muller NL, Heyneman L, Johkoh T, Park JS, Lee KS, Honda $\mathrm{O}$, Tomiyama N: Mycoplasma pneumoniae pneumonia: radiographic and high-resolution CT features in 28 patients. AJR Am J Roentgenol 2000, I74:37-4I.

13. Okada F, Ando Y, Wakisaka M, Matsumoto S, Mori H: Chlamydia pneumoniae and Mycoplasma pneumoniae pneumonia. Comparison of clinical findings and CT findings. J Comput Assist Tomogr 2005, 29:626-32.

14. Lee I, Kim TS, Yoon H-K: Mycoplasma pneumoniae pneumonia: CT features in 16 patients. Eur Radiol 2006, 16:719-25.

15. Nambu A, Saito A, Araki T, Ozawa K, Hiejima Y, Akao M, Ohki Z, Yamaguchi H: Chlamydia pneumoniae: comparison with findings of Mycoplasma pneumoniae and Streptococcus pneumoniae at thin-section CT. Radiology 2006, 238:330-8.

16. Miyashita N, Fukano H, Okimoto N, Hara H, Yoshida K, Niki Y, Matsushima $\mathrm{T}$ : Clinical presentation of community-acquired Chlamydia pneumoniae pneumonia in adults. Chest 2002, I21: I| 176-8I.

17. Ramirez JA, Ahkee S, Tolentino A, Miller RD, Summersgill JT: Diagnosis of Legionella pneumophila, Mycoplasma pneumoniae, or Chlamydia pneumoniae lower respiratory infection using the polymerase chain reaction on a single throat swab specimen. Diagn Microbiol Infect Dis 1996, 24:7-I4.

18. Miyashita N, Obase Y, Ouchi K, Kawasaki K, Kawai Y, Kobashi Y, Oka M: Clinical features of severe Mycoplasma pneumoniae pneumonia in adults admitted to an intensive care unit. J Med Microbiol 2007, 56: 1625-9.

19. Miyashita N, Fukano H, Yoshida K, Niki Y, Matsushima T: Is it possible to distinguish between atypical pneumonia and bacterial pneumonia?: evaluation of the guidelines for communityacquired pneumonia in Japan. Respir Med 2004, 98:952-60.

20. Reittner P, Ward S, Heyneman L, Johkoh T, Muller NL: Pneumonia: high-resolution CT findings in I /4 patients. Eur Radiol 2003, I3:5I5-2|.

21. Muller NL, Miller RR: Disease of the bronchioles: CT and histopathologic findings. Radiology 1995, I76:243-8.

22. Worthy SA, Muller NL: Small airway diseases. Radiol Clin North Am 1998, 36: $163-73$

23. Rollins S, Colby T, Clayton F: Open lung biopsy in Mycoplasma pneumoniae pneumonia. Arch Pathol Lab Med I986, I 1 0:34-4I.

24. Kadioglu A, Andrew PW: The innate immune response to pneumococcal lung infection: the untold story. Trends Immunol 2004, 25: 143-9.

25. Koletsky RJ, Weinstein A): Fulminant Mycoplasma pneumoniae infection. Report of a fatal case, and review of the literature. Am Rev Respir Dis 1980, I 22:491-6.

26. Chan ED, Welsh CH: Fulminant Mycoplasma pneumoniae pneumonia. West J Med 1995, 162:133-42.

\section{Pre-publication history}

The pre-publication history for this paper can be accessed here:

\section{http://www.biomedcentral.com/1471-2342/9/7/prepub}

